\title{
Developing a Public Health Framework for the Epidemiological Linkages between HIV/AIDS and NCDs: A Thematic Research Synthesis
}

\author{
Tilahun Nigatu, ${ }^{1, *}$, Geoffrey Sets we ${ }^{2}$, Julian Elliot ${ }^{3}$, B rian Oldenburg ${ }^{1}$ \\ ${ }^{1}$ Department of Epidemiology and Preventive Medicine, Monash University, Australia \\ ${ }^{2}$ School of Health Sciences, Monash University, South Africa \\ ${ }^{3}$ Infectious Disease Unit, Alfred Hospital, Australia
}

\begin{abstract}
The Political Declaration of High-level Meeting of the General Assembly on the Prevention and Control of NCDs has noted possible linkages between HIV/AIDS and NCDs. The aim of this study was to develop a public health framework for those linkages between HIV/AIDS and the common NCDs. The overall method used in this study was a thematic research synthesis. Descriptive, integrative and configurative syntheses of evidence on linkages between HIV/AIDS and NCDs were conducted. The searching process was iterative; appraisal was stepwise; and extraction was verbatim. All retrieved information was described, analysed and then configured in to a concept map using major pathways of relationships between HIV/AIDS and NCDs. The concept map was developed in to the public health framework through progressive review and revisions. Two major forms and pathways of lin kages between HIV/AIDS and NCDs were identified. There are five nodes that mediate the indirect HIV-NCD linkage: co mmon underlying factors, life style factors, antiretroviral treatment, common comp lications, and other mediating disease conditions. These lin kages along with med iating factors are organized in to a public health framework. In conclusion, the main pathways and forms of relationship between HIV/AIDS and NCDs were described; and a public health framework for the epidemiological interrelationships between HIV/AIDS and NCDs was developed.
\end{abstract}

Keywords Epidemiological Linkage, Public Health Framework, HIV/A IDS, NCDs

\section{Introduction}

After three decades in to the HIV epidemic, there are more than 33 million People living with HIV (PLHIV), two third of which are in sub-Saharan Africa. HIV/AIDS was a cause for about 1.8 million deaths in 2010. In the same year, 2.1 million new HIV infections have occurred. About $90 \%$ of these new HIV infections were in sub-Saharan Africa. Globally, the incidence of HIV has decreased by about $19 \%$ between 2001 and 2009. However, there is still dramatic variability in the change in incidence rate in specific settings[1]. About 7.2 million PLHIV are currently receiving antiretroviral treatment (ART). A lot has also been invested in the fight against this pandemic[2].

On the other hand, Noncommunicable diseases (NCDs) are the major killers worldwide. About 36 million of the 57 million total global deaths in 2008 were due to NCDs. NCDs cause three of the five deaths globally. The four common

* Corresponding author:

tilahun.haregu@monash.edu (Tilahun Nigatu)

Published online at http///journal.sapub.org/ijpt

Copyright (C) 2012 Scientific \& Academic Publishing. All Rights Reserved
NCDs, Cardiovascular diseases (CVD), cancers, chronic respiratory diseases (CRD), and Diabetes, account for about $80 \%$ of the total NCD deaths. The majority, $80 \%$, of the total NCD deaths occur in low-and-middle income countries. About $30 \%$ of NCD deaths are among people below 60 years of age[3]. The total NCD deaths are expected to reach 52 million if appropriate actions are not going to be taken. The four common NCDs share four common risk factors: unhealthy diet, insufficient physical activity, tobacco use and harmful use of alcohol.

The possible linkages between HIV/AIDS and NCDs are recognized at a higher level political declaration of the United Nations General Assembly on NCDs. The General assembly has acknowledged important linkages, associative relations, between the two disease conditions. These important linkages between HIV and NCDs are recommended for consideration, as appropriate, in the integration of health system responses to HIV/AIDS and NCDs[4]. The translation of these linkages in to the concept of integration of interventions needs public health models that illustrate broader perspectives of their relationships.

The interrelationship between HIV/AIDS and NCDs is complex and multidimensional. It also has various impacts 
on the development, progression and outcomes of both disease conditions. Nevertheless, there is minimal research documenting the impact and burden of common comorbidities of HIV/AIDS and NCDs. There are several studies that have examined the effect of antiretroviral treatments on the risk of developing NCDs. A few studies have also assessed the association between HIV infection itself and NCDs. Many other studies have reported factors that affect the risk for developing NCDs among PLHIV/AIDS who are receiving antiretroviral treatment [5-8].

While there has been some clinical comorbidity studies focusing on the relationship between HIV/AIDS and NCDs, there is no public health model for the complexities of HIV/AIDS and NCDs. In this regard, a public health framework can be an essential tool for research and programmatic activities involving HIV and NCDs. It guides the conceptualization of public health research, as well as the development of public health interventions for HIV/AIDS and NCDs by illustrating key themes and their interrelationships. It also depicts other possible factors that should be considered during the design and management of research and intervention programs. Thus, a public health framework for the linkages between HIV/AIDS and NCDs can be a foundation for the integration and interpretation of relevant information in the relationship between HIV/AIDS and NCDs [9-11]. In light of these, the aim of this study was to develop a public health framework for the epidemiological lin kages between HIV/AIDS and the four common NCDs.

\section{Methods}

\subsection{Search Strategy}

This study was a thematic synthesis of studies that describe the linkages between HIV/AIDS and NCDs. Any associative relationship between HIV/AIDS and NCDs was considered as a linkage. The search process was iterative. An initial search from simple electronic databases (Embase and Medline) was conducted to identify initial set of studies using the key words of HIV and at least one NCD term. Four studies were purposively selected for initial review.

After identification of initial themes from this review, a theme-based purposive searching of literature was conducted with the intention of maximizing heterogeneity of concepts. We aimed for purposive rather than exhaustive search as the purpose of the study was interpretive explanation and not prediction. Accordingly, more preference was given to recently published and review articles. We continued the iterative searching process till the point of 'conceptual saturation,' i.e. till no further new concept emerged.

\subsection{Appraisal}

We took the view that quality of the studies should be assessed before inclusion in to the review process. Accordingly, each artic le was assessed for its relevance to the major themes of the synthesis; reporting clarity of rationale, aims, methods, and findings; appropriateness of the methods used to assess the linkages between HIV/AIDS and NCDs; and added value of the study to the synthesis. These criteria were applied in a stepwise manner. Forty-eight studies that add new concepts or substantiate existing concepts were included in the synthesis.

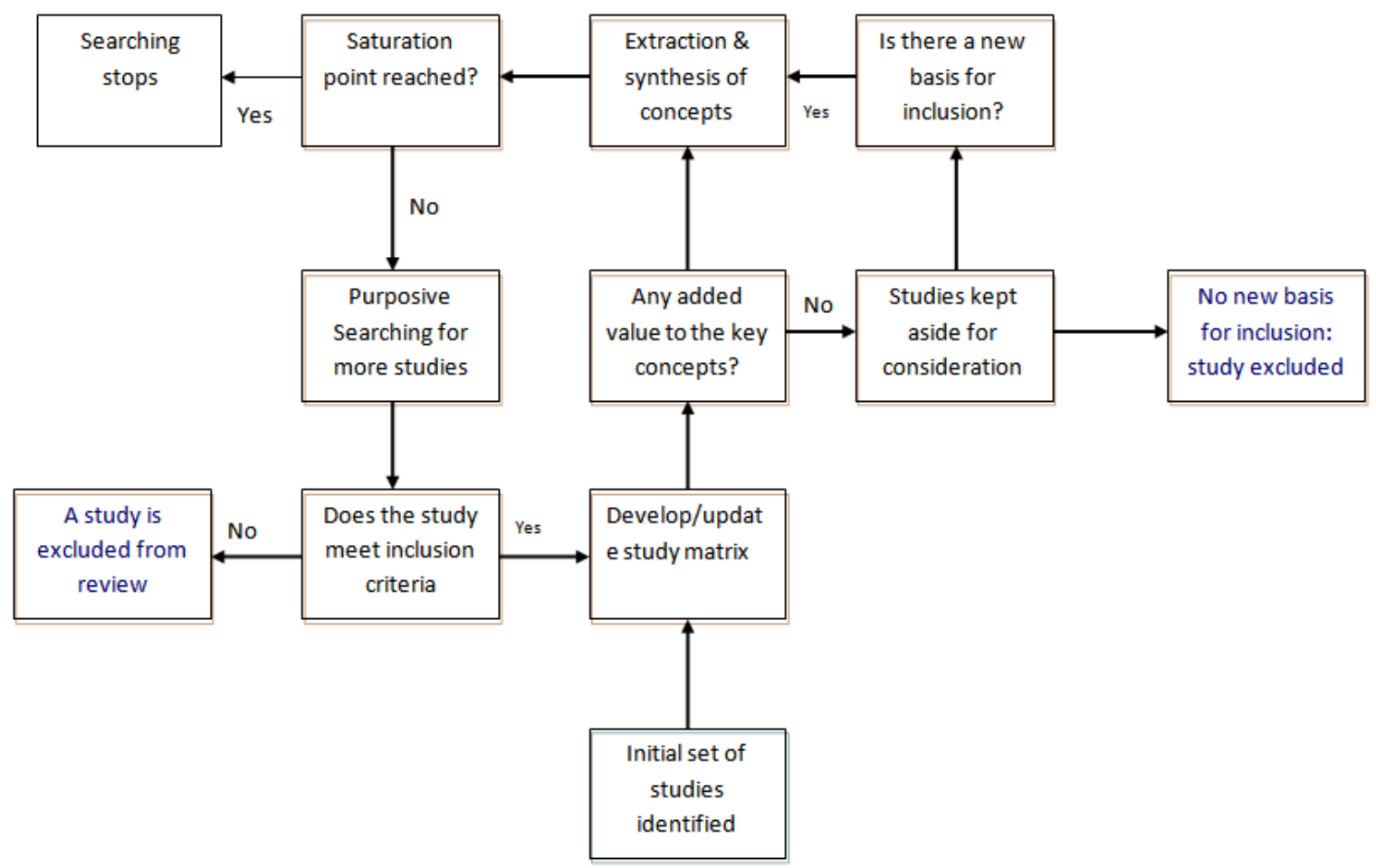

Figure 1. Flow chart of the searching, appraisal and extraction process 


\subsection{Data Extraction}

Information was ext racted fro $m$ the retrieved articles using a matrix that contains variables on the identifying information of the articles and the 'key concepts' documented by the article. These include the authors, title of the study, year of publication, study design, the type of NCD involved, study population, and 'key concepts' addressed by the article with regard to the linkage between HIV/AIDS and NCDs. Authors' own words were used in the extraction of key concepts-verbatim extraction. When extracting the key concepts, we have carefully reviewed abstract, result and conclusion sections of the studies. We extracted key concepts and imported then in to open code version 3.4 qualitative data analysis software program for coding and sorting (Figure 1).

\subsection{Synthesis of Information}

While this study is a thematic (qualitative) research synthesis in its overall design, it has used three key evidence synthesis approaches - descriptive, integrative, and configurative - to examine the interrelationships and to develop a public health framework that explains these interrelationships between HIV/AIDS and NCDs. We haven't considered these approaches to be sequential or experientially distinct.

A descriptive synthesis was conducted to aggregate relevant evidence that addressed similar descriptive themes of linkages between HIV/AIDS and NCDs. Key concepts were coded using broader categories, themes and subthemes. Information relevant to the interrelationship between HIV/AIDS and NCDs was sorted and summarized under different categories.

An integrative synthesis of the information summarized during the descriptive synthesis was the second form of synthesis in this study. The aggregated information was integrated in to major analytical themes and subthemes. These themes and subthemes were used to represent categories of information that address analytical concepts in the relationship between HIV/AIDS and NCDs. These themes were then formu lated in to forms and types of relationships.

Configurative synthesis (Framework development) was the third form of synthesis in this study. The themes and subthemes were configured in to a concept map, a diagram showing the relationships among concepts, which was used as a draft framework for displaying the relationships among the themes. Revision of the concept map was conducted through reflexive analysis of the evidence aggregated in the descriptive synthesis. This iterative and progressive approach has resulted in several versions of the draft public health framework. The final version of the framework was prepared after a series of reviews and revisions of the draft framework. The themes were considered as pathways of relationships among key variables in the framework.

\subsection{Presentation of Findings}

In the presentation of the findings, we have described the characteristics of the included studies that address concepts of linkage. We have also presented the analytical themes in the form of pathways of linkage and nodes that mediate the indirect pathways by citing the related studies. The public health framework that organizes all the information about HIV-NCD linkage is also included in the findings.

\section{Findings}

\subsection{Study Characteristics}

The studies included in this synthesis were published between 1990 and 2012. However, the majority of the studies [33/48] were published after 2005. Of the included studies, 10 mainly address direct linkage between HIV/AIDS and NCDs. The rest 38 mainly address indirect relationships between HIV and NCDs. With regard to the co mmon NCDs they mainly address, 27 studies address at least one of the four common NCDs as a primary consideration. By their design, about half of the studies were review articles. The rest were cohort, experimental and cross-sectional studies (Table 1).

Table 1. Characteristics of the studies included in the thematic synthesis

\begin{tabular}{|c|c|c|}
\hline Study characteris tics & Categories & $\begin{array}{c}\text { Number of } \\
\text { stu dies }\end{array}$ \\
\hline \multirow[t]{4}{*}{ Publication year } & $1990-1995$ & 2 \\
\hline & $1996-2000$ & 5 \\
\hline & $2001-2005$ & 8 \\
\hline & $2006-2012$ & 33 \\
\hline \multirow[t]{4}{*}{ Primary design } & Review articles & 28 \\
\hline & Experimental studies & 5 \\
\hline & Cohort studies & 8 \\
\hline & Cross-sectional studies & 7 \\
\hline \multirow{4}{*}{$\begin{array}{l}\text { NCD primarily ad- } \\
\text { dressed }\end{array}$} & Cardiovascular disease & 4 \\
\hline & Cancer & 7 \\
\hline & Chronic respiratory disease & 7 \\
\hline & Diabetes & 9 \\
\hline \multirow[t]{6}{*}{ Primary theme } & Direct HIV-NCD linkage & 10 \\
\hline & $\begin{array}{l}\text { Underlying/background } \\
\text { factors }\end{array}$ & 3 \\
\hline & Lifestyle factors & 7 \\
\hline & Antiret roviral treatment & 14 \\
\hline & Common complications & 5 \\
\hline & Other disease conditions & 9 \\
\hline
\end{tabular}

\subsection{Linkages between HIV/AIDS and NCDs}

Two major forms of relationships between HIV and NCDs emerged from the synthesis process. These were the determinant linkage, where the presence of one affects the 
probability of having the other, and the severity linkage, where the presence of one makes the progression and outcome of the other more or less worse. We have also identified two pathways of lin kage between HIV/AIDS and NCDs: Direct and indirect (Figure 2).

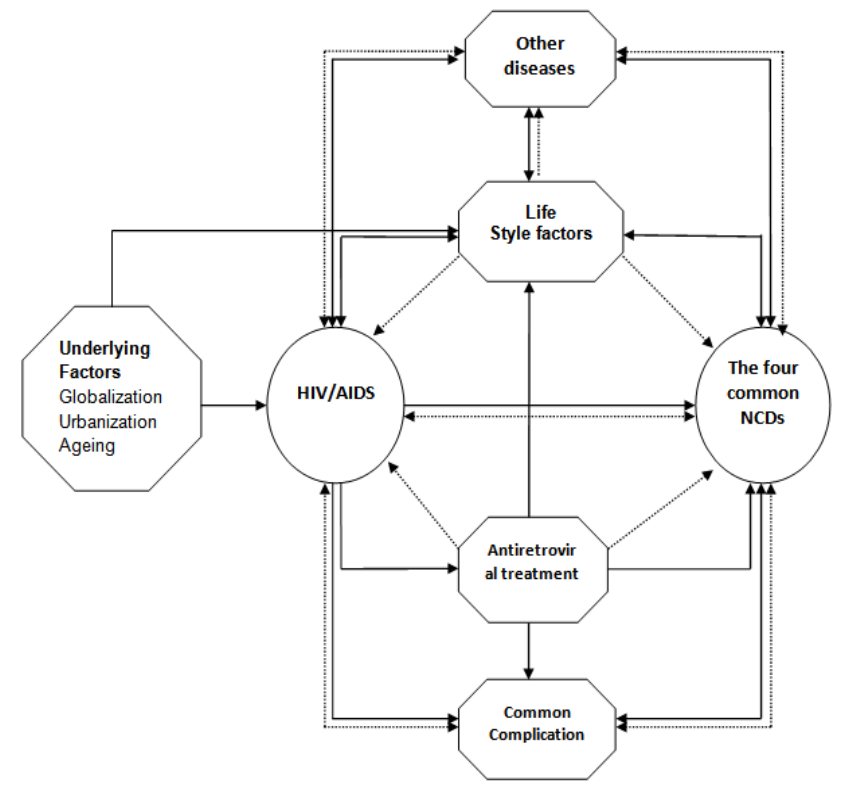

Note: Solid lines show determinant; broken lines show severity

Figure 2. Public Health framework for the possible linkages between HIV/AIDS and NCDs

\subsubsection{Direct Linkage between HIV/AIDS and NCDs}

HIV itself increases the risk of developing some NCDs. Studies have suggested that HIV-positive individuals have higher risk of developing common NCDs as compared to HIV-negative individuals[12]. People infected with HIV have higher rates of cancers, cardiovascular diseases and diabetes. A weakened immune system caused by HIV infection is a mong the contributing factors for the development of cancer in PLHIV[13]. HIV infection is also associated with low levels of HDL that increases the risk of cardiovascular diseases [14].

Even though some studies have suggested that HIV infection itself was not associated with increased risk of diabetes, others substantiate the evidence that insulin resistance may have an HIV disease associated component as well[ $[15$, 16]. Due to its autoimmune effect, untreated HIV could also be associated with higher risk of developing diabetes.

Type II diabetes could be a complication of untreated HIV infection; and effective viral suppression can reverse the disorder[17]. Other studies also support the association between HIV related lipodystrophy and insulin resistance. Treat ment of HIV re lated lipodystrophy with metformin may ameliorate insulin resistance[18]. This evidence suggested that diabetes could be a direct complication of HIV infection itself.

PLHIV have increased risk of CRD[19]. HIV in fection itself is an independent risk factor for CRD[20]. Although some of the increased risk of CRD among PLHIV can be attributed to smoking and drug abuse, the risk of CRD among this population group has remained high even after controlling the effect of these factors [21]. The increasing age among PLHIV due to treatment could also contribute to increased risk of CRD.

\subsubsection{Indirect Relationship between HIV/AIDS and NCDs}

The indirect relationship between HIV/AIDS and NCDs is mediated by five nodes: common underlying/background factors, life style factors, antiretroviral treatment, common complications, and other med iating disease conditions.

\section{Common underlying/background factors}

Major underly ing socio-economic, cultural, political and environmental determinants of chronic diseases, such as globalization, urbanization and ageing are common to both HIV and NCDs. Globalization and urbanization are the major driving factors for HIV/AIDS epidemic in low-and-middle income countries. Rapid changes in lifestyle due to the effects of the same factors are the major reasons for the emergence of NCDs in these countries[22, 23].

Due to the effect of these common underlying and background factors, HIV and NCDs are having an epidemiological convergence (Syndemicity) in low-and-middle income countries. As a result, urban areas in low-and-middle income countries have the highest share of both HIV and NCD burden. Hence, a macro-level relationship between HIV/AIDS and NCDs could be better explained by these common underlying and background factors.

Along with the traditional life style related risk factors of NCDs that emanate fro $m$ the underlying socio-economic and cultural factors, ageing of PLHIV as a result of the intended effect of HIV treatment is also among the factors that increase the predisposition of PLHIV to a higher risk of NCDs like Diabetes[24]. On the other hand, recent evidence shows that NCDs in the general population tend to occur at earlier age than normally expected. Early onset NCDs that occur before 60 years of age is presumably higher in low and middle income countries where the prevalence of HIV has been high for decades.

\section{Lifestyle factors}

HIV/AIDS and HIV treatment are associated with changes in life style factors such as dietary habits and physical exercise that influence the risk of developing NCDs among PLHIV[25]. Concerns revolving around the belief that physicale xerc ise could result in decrease effectiveness of the immune system and low CD4 cell count, despite the existence of strong evidences against such belief, are still common in low-and-middle inco me countries and could possibly affect the physical activity pattern of a person living with HIV[26].

Two common body changes associated with HIV/AIDS are lipodystrophy and wasting syndrome. These changes are man ifested by loss of fat in the face, arms, legs and buttocks and gain of fat in the abdomen, back of the neck and breasts. More than the usual but carefully planned exercise is needed to prevent the effects of these body changes. The absence of 
the required level of physical exercise may increase the risk of high cholesterol, triglycerides and blood glucose levels [27]. These metabolic disorders are the major causes of cardiovascular diseases and diabetes.

PLHIV may require mod ifications in theird ietary habits in order to protect themselves from the dangers of HIV and HIV treatment. While the intake of high quality diet may help slow the progressive HIV infection and prolong life, the intake of high calorie diet could increase blood cholesterol and blood sugar levels as a result of the metabolic changes that occur along with lipodystrophy such as insulin resistance and dyslipidaemia. Therefore, both the amount and kind of food are equally important in dietary modifications associated with HIV/AIDS[28].

Malnutrition, in areas where HIV prevalence is high and food insecurity is endemic, has multiple effects on the immune system such as decrease in CD4 cell count, suppression of delayed hypersensitivity and production of abnormal $\beta$-cells, a condition leading to the development of diabetes. The immune suppression effect of malnutrition is similar to that of the HIV infection itself[29-31].

The effect of antiretroviral treatment on the risk of developing NCDs

Quite a lot of research confirmed that some antiretroviral drugs increase the risk of cardiovascular diseases and Diabetes $[6,32,33]$. Low HDL cholesterol levels may be caused by ART-associated lipodystrophy[34]. Different possible mechanis ms of insulin resistance and diabetes in HIV infection has been pointed out[7, 34]. However, the actual mechanis ms by which antiretroviral drugs increase the risk of developing these diseases are not clearly established though several possible pathways have been postulated[34].

The risk of antiretroviral drugs related cardiovascular diseases and diabetes depends on the type of the drugs used, the duration of treatment, and other patient related factors. In this regard, the use of protease inhibitors and longer duration of exposure to antiretroviral treatment have strong association with higher risk of these diseases. There is also a hypothesis that the recovery of the human immune system immediately after initiation of antiretroviral treatment may predispose a person to autoimmune disease in the form of type 1 diabetes [27, 35].

On the other hand, antiretroviral treatment is associated with decreased risk of cancer in PLHIV. The incidence of both AIDS-related cancers and non-AIDS-related cancers was found to be lower among those PLHIV who have started ART[36, 37, 38]. This might be due to the effect of the treatment in suppressing HIV replication and thereby reconstituting the immune system. There is also suggestive evidence that some ARV drugs may have antitumor effect[39].

Studies addressing the relationship between antiretroviral treatment and CRD indicate that airway obstruction may be one of the latest complications lin ked to A RT. Explanations include direct drug effect, restoration of the immune system and development of autoimmun ity $[40,41]$.

Common complications
Besides the proposition that NCDs can be a direct complication of untreated HIV infection and antiretroviral treatment, HIV/AIDS and NCDs share some important common complications. Among these are metabolic, neurologic, cardiovascular, and renal complications[42]. For instance, albuminuria, which is a key marker of kidney disease, can be a complication of both HIV and Diabetes. Evidence has shown that the prevalence of albuminuria among people with both HIV and diabetes was twofold greater than those with either disease alone[43]. Th is was attributed to the additive effect of the impacts of the two diseases. Kidney diseases are also associated with some cardiovascular diseases. Treatment with combined ART in PLHIV can also induce several metabolic complications like lipodystrophy, and dyslipidaemia. The same complications can also result from poorly managed diabetes. While cardiovascular diseases can be a complication of diabetes, they also share common metabolic complication with diabetes.

There are also some neurological comp lications which are common to HIV, antiretroviral treatment and NCDs. Neurologic complications are also as sociated with cardiovascular diseases [44]. Neurological complications such as stroke, nerve damage and cognitive impairment can also be complications of poorly managed Diabetes too[45]. CRD can increase the risk of pulmonary infections and cardiovascular diseases, both of which are also complications of HIV infection[56].

Cancers also have some cardiovascular and metabolic complications that are also complications of the HIV infections and antiretroviral treatment. The relationship between $\mathrm{HIV}$ and NCDs through common complications is very complex. But several studies have generated evidence that affirm the relationship between HIV, ART and NCDs via common complications.

Other disease conditions

As both HIV/AIDS and NCDs are related to several other disease conditions, which may complicate or be a comp lication of them, the relationship between HIV/AIDS and NCDs can be mediated by several other disease conditions.

Cancer-related viruses such as Human herpsvirus 8 (HHV-8), Human Papillo ma virus (HPV), Epsteinbarr virus (EBV), hepatitis B virus (HBV) and Hepatitis C virus (HCV) are more common among PLHIV. As HIV affects the body's ability to fight these infections, cancers related to these viruses are higher among PLHIV[47, 48]. HCV is also related with Diabetes. HCV infection prevalence may reach $40 \%$ among HIV positive people in some settings. HCV infection has many risk factors in co mmon with HIV. The HCV load is usually higher in HIV co-in fected people than people with HCV alone. As HCV affects the liver, it increases the risk of developing diabetes among PLHIV[49]. This makes HCV infection to be one of the mediating factors in the relationship between HIV/AIDS and NCDs.

Tuberculosis (TB) is associated with HIV, Diabetes and CRD. As a result of the HIV-associated weakening of the immune system, HIV-positive people have higher rates of Tuberculosis, as compared to HIV-negative individuals [50]. 
People with Diabetes also have an increased risk of Tuberculosis[51]. Studies have indicated that Diabetes and TB epidemics are now converging. The prognosis among patients with both diseases is poor. Some studies went further and considered the possibility that tuberculosis may cause Diabetes[52]. TB is also associated with CRD. CRD patients have a higher risk of developing active TB compared to the general population[53]. Hence TB is related with both HIV and CRD and acts as a linking factor between the two diseases.

Another important issue is the relationship between vitamin D Deficiency and Diabetes in HIV infection[54]. The presence of vitamin D deficiency is associated with poor health outcomes in PLHIV. Some ARV drugs, like Nevirapin, also lower the concentration of vitamin $\mathrm{D}$ in the body. Vitamin D deficiency is also associated with cardiovascular disease[55]. Though there is limited evidence on the underlying mechanis ms, vitamin D deficiency could be one of the mediating factors in the epidemiological linkage between HIV/AIDS and NCDs.

\section{Discussion}

This synthesis has outlined the major forms and pathways of epidemiological linkages between HIV and NCDs. The linkages are related to stages of development, progression, management and outcomes of HIV/AIDS and NCDs. These links provide a basis for integrated prevention and management of HIV and NCDs. Understanding these interrelationships between HIV and NCD also helps the management of complications of the two disease conditions. It will also facilitate integration of HIV and NCD programs [56].

The public health framework developed in this study is expected to be an important input to public health researches that address the intersection of HIV/AIDS and NCDs. It will help locate where a research theme lies within the broader framework of the relationship between HIV/AIDS and NCDs.

This public health framework integrates the various interrelationships between HIV/AIDS and NCDs in the course of development, progression and outcome. This knowledge is important for coord inated approaches in the provision of HIV/AIDS and NCDs prevention, treatment and care services.

Fro $m$ the perspective of program design, implementation and evaluation, the public health framework developed in this study could be a useful guide at allstages. In the problem analysis phase of HIV/AIDS and/or NCD program development, this public health framework can inform program development experts about important aspects that should be considered in the program package.

Understanding the relationship between HIV and NCDs also fosters the integration of services and consideration of HIV-NCD comorbidity during program implementation. During program evaluation, the public health framework could help in formulating appropriate evaluation questions and developing applicable evaluation tools. It will also help in interpreting and attributing evaluation findings fro $\mathrm{m} \mathrm{HIV}$ and NCD programs.

In this period of high political co mmitment devoted to the prevention and control of both NCDs and HIV/AIDS, this public health framework is expected to be a basis for more comprehensive chronic disease prevention and control approaches in future policy decisions.

However, this study had some limitations. There was some conflicting evidence encountered in the analysis of the relationship between HIV/AIDS and NCDs. Those relationships with contradicting evidence were considered to be possible and are included in the framework. Information from single case studies is considered to be suggestive rather than conclusive. Second, this public health framework was developed based on epidemiological associations between HIV and the four common NCDs. Thus, it may not reflect cause-and-effect relationships.

In conclusion, this study has pointed out possible linkages between HIV/AIDS and NCDs. The public health framework illustrates these important interrelationships. Though such a framework may not fit all conditions, it is expected to serve as a generic framework for visualizing major issues in the relationship between HIV/AIDS and NCDs. The framework should be adapted to specific contexts before application for research and intervention programs.

\section{REFERENCES}

[1] UNAIDS. UNAIDS report on the Global AIDS Epidemic 2010. Available at: http://www.unaids.org/globalreport/docu ments/20101123_GlobalReport_full_en.pdf. Accessed on June 6, 2012

[2] US PEPFAR. PEPFAR Funding: Investments that save lives and promote security. Available at: http://www.pep far.gov/d ocuments/organization/80161.pdf. Accessed on June 6, 2012

[3] World Health organization. Global Status report on Noncommunicable diseases 2010. Available at: http://www.who.int/nmh/publications/ncd_report_full_en.pd f. Accessed on June 6, 2012

[4] United Nations General Assembly. Political declaration of the High-level Meeting of the General Assembly on the Prevention and Control of Non-communicable Diseases. $66^{\text {th }}$ session, Sept 2011. Paragraph 27

[5] De Wit, S., et al., Incidence and risk factors for new-onset diabetes in HIV-infected patients. Diabetes Care, 2008. 31(6): p. 1224-1229.

[6] Brown, T.T., et al., Antiretroviral therapy and the prevalence and incidence of diabetes mellitus in the multicenter AIDS cohort study. Archives of Internal Medicine, 2005. 165(10): p. $1179-1184$

[7] Ledergerber, B., et al., Factors associated with the incidence of type 2 diabetes mellitus in HIV-infected participants in the Swiss HIV cohort study. Clinical Infectious Diseases, 2007. 45(1): p. 111-119. 
[8] Tien, P.C., et al., Antiretroviral therapy exposure and incidence of diabetes mellitus in the Women's Interagency HIV Study. AIDS, 2007. 21(13):p. 1739-1745

[9] Banzi, R., et al., Conceptual frameworks and empirical approaches used to assess the impact of health research: An overview of reviews. Health Research Policy and Systems, 2011.9.

[10] Coen, S.E., et al., A relational conceptual framework for multidisciplinary health research centre infrastructure. Health Research Policy and Systems, 2010. 8.

[11] Kagan, J.M., et al., Developing a conceptual framework for an evaluation system for the NIAID HIV/AIDS clinical trials networks. Health Research Policy and Systems, 2009. 7.

[12] Hommes, M.J.T., et al., Insulin sensitivity and insulin clearance in human immunodeficiency virus-infected men. Metabolism: Clinical and Experimental, 1991. 40(6): p. 651-656.

[13] Grulich, A.E., et al., Incidence of cancers in people with HIV/AIDS compared with immunosuppressed transplant recipients: a meta-analysis. Lancet, 2007. 370(9581): p. 59-67.

[14] Mujawar, Z., et al., Human immunodeficiency virus impairs reverse cholesterol transport from macrophages. PLoS biology, 2006. 4(11): p. e365.

[15] Butt, A.A., et al., HIV infection and the risk of diabetes mellitus. AIDS, 2009. 23(10): p. 1227-1234.

[16] Dube, M.P., Disorders of glucose metabolism in patients infected with human immunodeficiency virus. Clinical infectious diseases: an official publication of the Infectious Diseases Society of America, 2000. 31(6): p. 1467-1475.

[17] Koeppe, J. and L. Kosmiski, Apparent resolution of type 2 diabetes mellitus after initiation of potent antiretroviral therapy in a man from Africa with HIV infection. Clinical infectious diseases: an official publication of the Infectious Diseases Society of America, 2006. 42(10): p. e79-81.

[18] Mikhail, N., Insulin resistance in HIV-related lipodystrophy. Current Hypertension Reports, 2003. 5(2): p. 117-121.

[19] Crothers, K., Chronic Obstructive Pulmonary Disease in Patients Who Have HIV Infection. Clinics in Chest Medicine, 2007. 28(3): p. 575-587.

[20] Diaz, P.T., et al., Increased susceptibility to pulmonary emphysema among HIV-seropositive smokers. Annals of Internal Medicine, 2000. 132(5): p. 369-372.

[21] Crothers, K., et al., Increased COPD among HIV-positive compared to HIV-negative veterans. Chest, 2006. 130(5): p. 1326-1333.

[22] Magnusson, R.S., Non-communicable diseases and global health governance: Enhancing global processes to improve health development. Globalization and Health, 2007. 3.

[23] Coovadia, H.M. and J. Hadingham, HIV/AIDS: Global trends, global funds and delivery bottlenecks. Globalization and Health, 2005. 1.

[24] Schmid, G.P., et al., The unexp lored story of HIV and ageing. Bulletin of the World Health Organization, 2009. 87(3): p. 162-162A.

[25] Hadigan, C., Dietary habits and their association with metabolic abnormalities in human immunodeficiency virus-related lipodystrophy. Clinical Infectious Diseases, 2003. 37(SUPPL. 2): p. S101-S104.

[26] Bopp, C.M., et al., Clinical implications of therapeutic exercise in HIV/AIDS. The Journal of the Association of Nurses in AIDS Care : JANAC, 2003. 14(1): p. 73-78.

[27] Jones, S.P., et al., Short-term exercise training improves body composition and hyperlipidaemia in HIV-positive individuals with lipody strop hy. AIDS, 2001. 15(15):p. 2049-2051.

[28] Ahoua, L., et al., Nutrition outcomes of HIV-infected malnourished adults treated with ready-to-use therapeutic food in sub-Saharan Africa: a longitudinal study. Journal of the International AIDS Society, 2011. 14: p. 2.

[29] Gorbach, S.L., T.A. Knox, and R. Roubenoff, Interactions between nutrition and infection with human immunodeficiency virus. Nutrition Reviews, 1993. 51(8): p. 226-234.

[30] Scrimshaw, N.S. and J.P. SanGiovanni, Synergism of nutrition, infection, and immunity: An overview. American Journal of Clinical Nutrition, 1997. 66(2): p. 464S-477S.

[31] Beisel, W.R., Nutrition and immune function: Overview. Journal of Nutrition, 1996. 126(10 SUPPL.): p. 2611S-2615S.

[32] Grinspoon, S. and A. Carr, Cardiovascular risk and body-fat abnormalities in HIV-infected adults. New England Journal of Medicine, 2005. 352(1): p. 48-62.

[33] Carr, A. and D. Ory, Does HIV cause cardiovascular disease? PLoS Medicine, 2006. 3(11): p. 1986-1987.

[34] Das, S., Insulin resistance and diabetes in HIV infection. Recent Patents on Anti-Infective Drug Discovery, 2011. 6(3): p. $260-268$.

[35] Takarabe, D., et al., Autoimmune diabetes in HIV-infected patients on highly active antiretroviral therapy. Journal of Clinical Endocrinology and Metabolism, 2010. 95(8): p. 4056-4060.

[36] Engels, E.A., et al., Cancer risk in people infected with human immunodeficiency virus in the United States. International Journal of Cancer, 2008. 123(1): p. 187-194.

[37] Engels, E.A., et al., Trends in cancer risk among people with AIDS in the United States 1980-2002. AIDS, 2006. 20(12): p. $1645-1654$

[38] Silverberg, M.J. and D.I. Abrams, AIDS-defining and non-AIDS-defining malignancies: Cancer occurrence in the antiretroviral therapy era. Current Opinion in Oncology, 2007. 19(5): p. 446-451.

[39] Monini, P., et al., Antitumour effects of antiretroviral therapy. Nature Reviews Cancer, 2004. 4(11): p. 861-875.

[40] George, M.P., et al., Respiratory symptoms and airway obstruction in HIV-infected subjects in the HAART era. PLoS ONE, 2009. 4(7).

[41] Gingo, M.R., et al., Pulmonary function abnormalities in HIV-infected patients during the current antiretroviral therapy era. American Journal of Respiratory and Critical Care Medicine, 2010. 182(6): p. 790-796.

[42] Bader, M.S. and D.V. Kelly, Diagnosis and management of common chronic metabolic complications in HIV-infected 
patients. Postgraduate Medicine, 2008. 120(4): p. 17-27.

[43] Kim, P.S., et al., Increased prevalence of albuminuria in HIV-infected adults with diabetes. PLoS ONE, 2011. 6(9).

[44] Cheung, R.T.F., Neurological complications of heart disease. Bailliere's Clinical Neurology, 1997. 6(2): p. 337-355.

[45] Flower MJ. Micro vascular and Macro vascular Complications of Diabetes. Clinical Diabetes 2008; 26 (2): 77-82

[46] Gale, N.S., et al., Does pulmonary rehabilitation address cardiovascular risk factors in patients with COPD? BMC Pulmonary Medicine, 2011. 11.

[47] Powles, T., et al., Hepatocellular cancer in HIV-infected individuals: Tomorrow's problem? Expert Review of Anticancer Therapy, 2006. 6(11): p. 1553-1558.

[48] Chaturvedi, A.K., et al., Risk of human papillomavirus-associated cancers among persons with AIDS. Journal of the National Cancer Institute, 2009. 101(16): p. 1120-1130.

[49] Jain, M.K., et al., Hepatitis C is associated with type 2 diabetes mellitus in HIV-infected persons without traditional risk factors. HIV Medicine, 2007. 8(8): p. 491-497.
[50] Datiko, D.G., et al., The rate of TB-HIV co-infection depends on the prevalence of HIV infection in a community. $B M C$ public health, 2008. 8.

[51] Jeon, C.Y. and M.B. Murray, Diabetes mellitus increases the risk of active tuberculosis: A systematic review of 13 observational studies. PLoS Medicine, 2008. 5(7): p. 1091-1101.

[52] Szep, Z., et al., Vitamin D deficiency is associated with type 2 diabetes mellitus in HIV infection. AIDS, 2011. 25(4): p. 525-529.

[53] Inghammar, M., et al., COPD and the risk of tuberculosis - a population-based cohort study. PLOS ONE, 2010. 5(4).

[54] Dooley, K.E. and R.E. Chaisson, Tuberculosis and diabetes mellitus: convergence of two epidemics. The Lancet Infectious Diseases, 2009. 9(12): p. 737-746.

[55] Wang, T.J., et al., Vitamin D deficiency and risk of cardiovascular disease. Circulation, 2008. 117(4): p. 503-511.

[56] UNAIDS. Chronic care of HIV and Noncommunicable diseases: How to leverage the HIV experience. UNAIDS report 2010. 